\title{
A New Type Proton Exchange Membrane Preparation of Doping Phosphotungstic Acid in High Temperature and Low Humidity
}

\author{
Ruizhe Wang ${ }^{1}$ \\ ${ }^{1}$ School of Electrical and Electric Engineering, North China Electric Power University, Beijing, \\ 102206, China
}

Keywords: PHNT, Proton Exchange Membrane, Conductivity

\begin{abstract}
The energy of a proton exchange membrane fuel cell (PEMFC) as a pollution-free and efficient conversion device has broad application prospects in the new energy vehicles and other aspects. Improving PEMFC operating temperature is the main research directions. Proton exchange membrane, one of the key material of the PEMFC determines the operating temperature of the PEMFC. By mixing the proton exchange membrane with phosphotungstic acid(HPW) or other heteropolyacids with high proton conductivity and boiling point, can effectively enhance the membrane water retention to improve proton exchange membrane's conductivity at high temperature and low humidity.

The main content of this paper is to prepare a new proton exchange membrane mixed with HPW and PHNT based on SPEEK. By adjusting the distribution of SPEEK, PHNT and HPW and taking proton exchange membrane characterization tests to obtain. Theoptimum distribution group's conductivity compared to pure SPEEK proton exchange membrane prepared has significantly increased by $36.39 \%$.
\end{abstract}

\section{Background}

As a clean and efficient energy conversion devices, proton exchange membrane fuel cell (PEMFC) has broad application prospects in the new energy vehicles, distributed power station, mobile power, national defense and military aspects. However, PEMFC study exists these issues currently such as complex management of battery thermal water, easy to be catalyst poisoned, low catalytic efficiency and so on. Improving PEMFC operating temperature is one of the effective measures to solve these problems, and accelerate the battery cathode oxygen reduction reaction in particular based on dynamics to improve the efficiency of the battery. Therefore, high-temperature PEMFC technology has become the main direction of today's fuel cell development.

As one of the key materials, proton exchange membrane determines the operating temperature of the PEMFC operating temperatures ${ }^{[1]}$. Due to the water as Nafion proton conducting medium, conventional perfluorosulfonic acid type Nafionmembranes has the operating temperatureunder 80 degrees Celsius. When the battery temperature exceeds 100 degrees Celsius, proton membrane evaporation of water will result in a sharp decline in performance of proton conductivityand prone to structural changes, chemical degradation and decreasedmechanical properties at high temperature. For these reasons, high-temperature proton exchange membrane research and development has been taken widespread attention, the focus of research focused on how to improve the proton conductivity under high temperature conditions in order to meet the normal conduction properties of efficient fuel cell operation. 


\section{Experimental Content}

By mixing the proton exchange membrane with Phosphotungstic acid or other heteropolyacids with high proton conductivity and boiling point, can effectively improve proton exchange membrane's conductivity.However, the phosphotungstic acid is easily soluble in water, there is no good way to effectively prevent the loss of phosphotungstic acid during cell operation from the proton exchange membrane. This project intends to uses synthetic graft based on polydopamine and basic polymer chain to modified halloysite nanotube surface. The use of acid-base pairs acts to stabilize phosphotungstic acid, andutilizing "capillary action" within the halloysite nanotube to further enhance moisture retention, enabling to improve the composite proton exchange membrane at high temperature and low humidity proton conductivity. implementation of the project will provide new ideas for preparation and properties of novel composite proton exchange membrane.

Experiments can be broadly divided into five steps.

(1)Doing polydopaminemodified for halloysite nanotubes(HNT) to get DHNT.

(2)Connecting the vinyl pyrrolidone (PVP) in the DHNT surface by ATRP modified reaction to obtain a basic polymer chain to get PHNT.

(3) Preparingcomposite proton exchange membrane based on these two rhetorical halloysite nanotubes (PHNT) and doped with phosphotungstic acid (HPW).

(4)Doing structural characterization for experimental obtained DHNT and PHNT including infrared spectroscopy test, transmission electron microscopy and chemical elemental analysis to determine the effect of experimental graft.

(5)Doing tests for finally obtained proton exchange membrane, including scanning tunneling microscopy, water absorption and conductivity testing to determine the effect of the membrane preparedand comparingwith pure SPEEKto test the membrane's conductivity actual improvement.

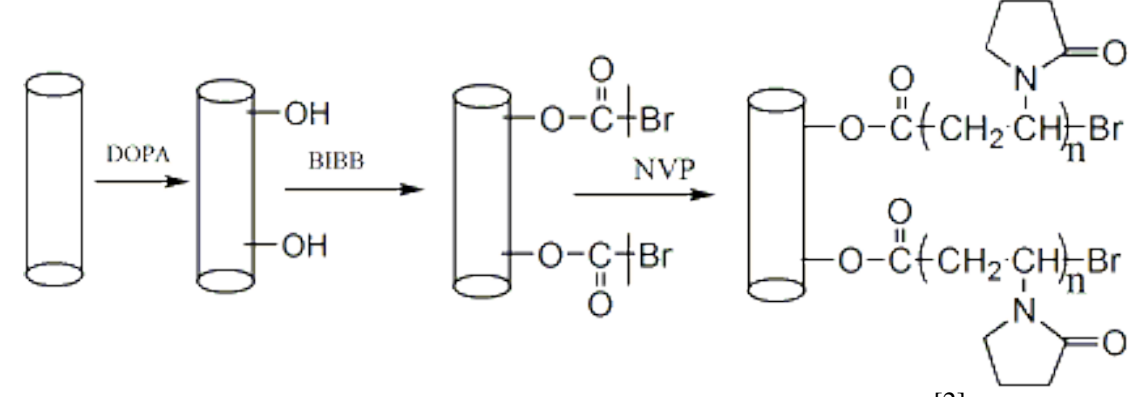

Figure 1Technical route modification experiments ${ }^{[2]}$

\section{Research Results and Conclusions}

Characterization of Modified Halloysite. We do polydopamine modified to get DHNT on halloysite nanotubes. Based on this conduct ATRP reaction, generating a basic group of polyvinylpyrrolidone (PVP) to get PHNT, and which were characterized.

TEM Observe. After a good preparation ofPHNT and DHNT, we observe comparatively of the microstructure DHNT and PHNT (as shown in Figure 2) through the use of FEI's Quanta 200F field emission scanning electron microscopy. PHNT nano tube diameter is smaller than DHNT's as it can be seen in DHNT and PHNT SEM figure. We can conclude that when conducting ATRP reaction, DHNT does have some substance connected to the nanotubes. 


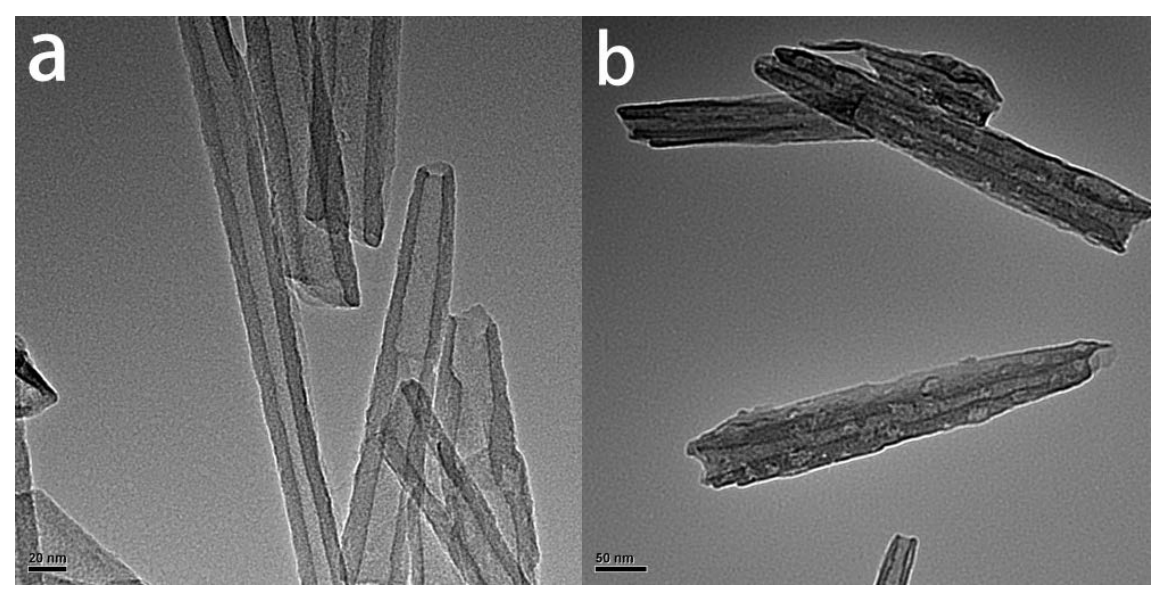

Figure 2DHNT(a)与 PHNT(b)'s TEM FIG.

Infrared Spectroscopy. From the Figure 3 below, we compare the DHNT (after polydopamine modified halloysite) and PHNT (after PVP modified DHNT). Wherein, there both have a nearby peak at a wavelength of $1700 \mathrm{~cm}-1$. PHNT absorption peak has some more. So we can come to a conclusion that higher PHNT carbonyl takes higher proportion compared with DHNT, and vinylpyrrolidonecontains a carbonyl group in its structure ${ }^{[3]}$, which preliminary describesvinylpyrrolidone grafted on DHNT in ATRP modification reaction.

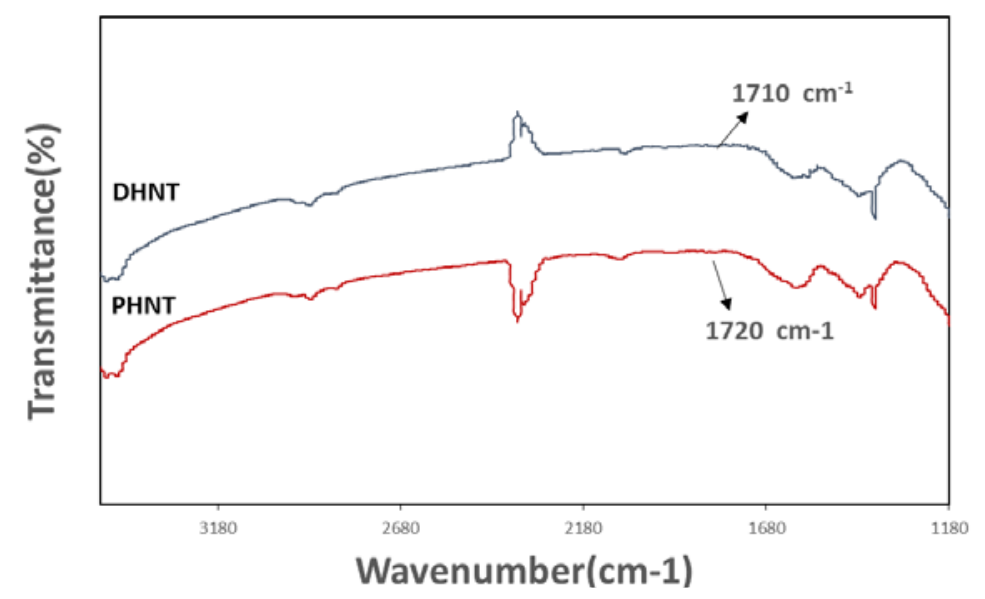

Figure 3 DHNT andPHNT'S IRanalysiscurve

Chemical Element Analysis. Chemical element analysis is to use chemical element analyzer to measure DHNT, PHNT's N, C, H, S and other elements. the type of material can be determined by analyzing element mass proportion, The organic elemental analysis can be shown in Table 1 and Table 2 by using the instrument model VarioELcube.

Table 1Elemental analysis data one

\begin{tabular}{lcccl}
\hline $\begin{array}{l}\text { Measuring } \\
\text { material }\end{array}$ & $\mathrm{N} \mathrm{[ \% ]}$ & $\mathrm{C} \mathrm{[ \% ]}$ & $\mathrm{H}[\%]$ & $\mathrm{S}[\%]$ \\
\hline DHNT & 0.011 & 0.032 & 0.973 & 0.013 \\
PHNT & 1.211 & 7.771 & 1.133 & 0.01 \\
\hline
\end{tabular}

Table 2Elemental analysis data two

\begin{tabular}{llll}
\hline projects & $\mathrm{N}(\%)$ & $\mathrm{C}(\%)$ & $\mathrm{C}: \mathrm{N}$ \\
\hline PHNT-DHNT & 1.2 & 7.739 & $6.1: 1$ \\
\hline
\end{tabular}

By contrast C/N ratio in NVP and PHNT-DHNT, we can confirme PVP grafted in ATRP reaction. Because polydopamine has carbon and nitrogen element, $\mathrm{C} / \mathrm{N}$ ratio will be some errors. The carbon 
mass conservation element can be obtained by the following Equation (3-1).

$$
\frac{m_{p v p} \times 64.78 \%}{m_{P H N T}}=7.771 \%(3-1)
$$

So we can calculate PVP taking accounted for $12.0 \%$ in the PHNT. Based on the above analysis, it can be further confirmed that PVP are grafted in ATRP reactions.

Characterization of Proton Exchange Membrane. According to the results of our previous experiments, which compared to pure SPEEK proton exchange, the group of conductivity improvinghighest is 6: 1: 3 (in accordance with SPEEK: PHNT: proportion HPW). Based on this result, we developed a new recipe: pure SPEEK, 6:0:4, 6:4:0, 6:1:3, 5:1.25:3.75, 7:0.75:2.25, 6:2:2, 6:0.8:3.2, nafion and we prepare to get membrane.

Scanning Electron Microscope Observe. After the good preparation for nine membranes, we choose pure SPEEK proton exchange membrane (numbered a), (phosphotungstic acid) SPEEK proton exchange membrane added HPW (number b, SPEEK: HPW = 6: 4), SPEEK proton exchange membrane plus PHNT (numbered c, SPEEK: PHNT = 6: 4), SPEEK proton exchange membrane added PHNT and HPW (number d, SPEEK: PHNT: HPW = 6: 2: 2), these four membranesto do scanning electron microscope test(Figure 4). In the figure 4, we can clearly see the shape and distribution of the nanotubes in the number $\mathrm{c}$ and $\mathrm{d}$ compared with number a, which shows PHNT in SPEEK membrane dispersing well.

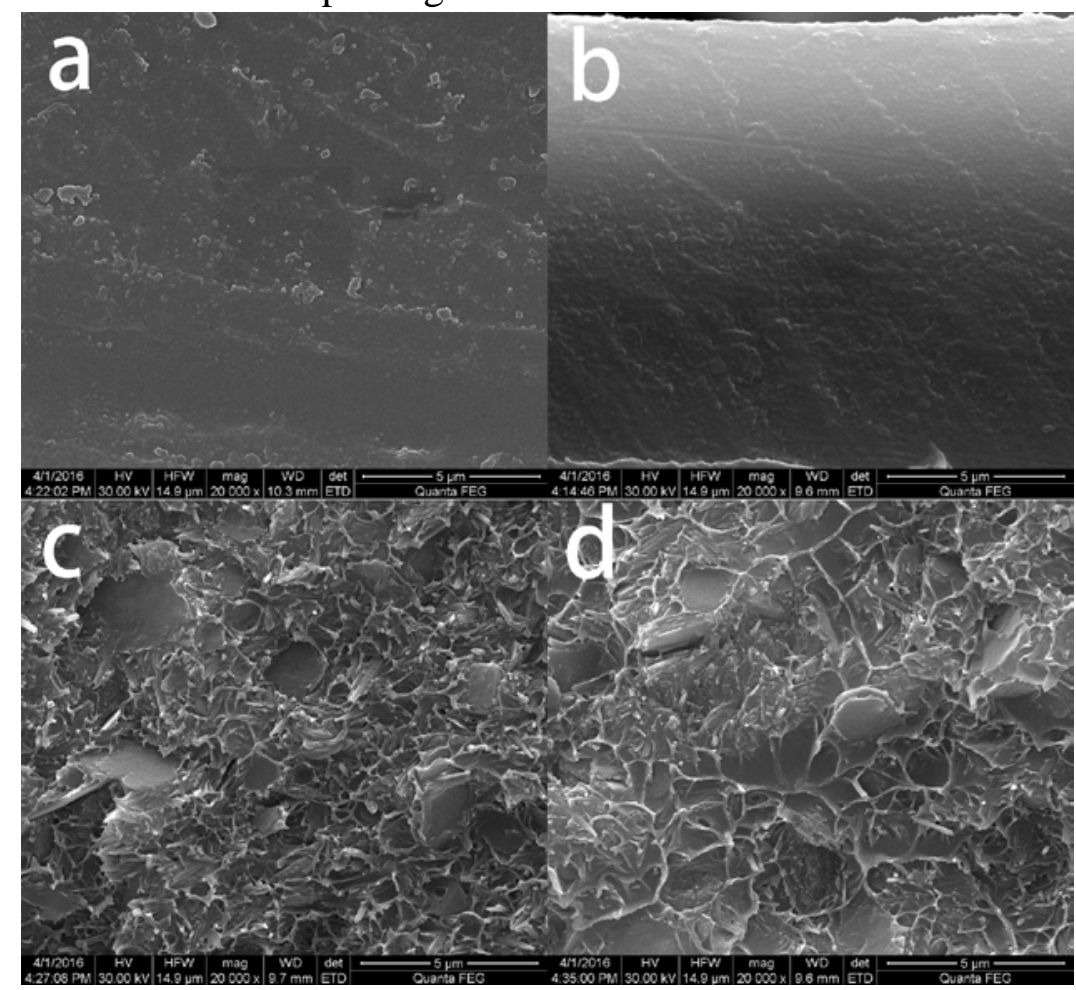

Figure 4Scanning Electron Microscope Fig

(a) SPEEK (b) SPEEK:HPW=6:4（c） SPEEK:PHNT=6:4 （d） SPEEK:PHNT:HPW=6:2:2

Conductivity Test. By testing each piece of membrane's conductivity, the resulting data collected are summarized in Figure 5. Seen from the final result, we made the membrane, the number c (SPEEK: PHNT: HPW = 6: 1: 3) and the number d (SPEEK: PHNT: HPW = 5: 1.25: 3.75) compared to the number a (pure SPEEK), the conductivity was increased by $34.24 \%$ and $36.39 \%$, compared to the number b (SPEEK: PHNT = 6: 4), the efficiency is improved by 2.4 times. This shows that the proportion of modified halloysite and phosphotungstic acid in 1: 3 is about the best ratio accounting for around $60 \%$ of SPEEK. Furthermore, number b has less efficient shown in Fig 
5. One reason is nophosphotungstic acid was added, the other is PHNT taking a large proportion causing nouniformdispersion. Thus, the proportion of PHNT shouldn't too much. Meanwhile, the proportion phosphotungstic acid should be increase appropriately in the membrane.

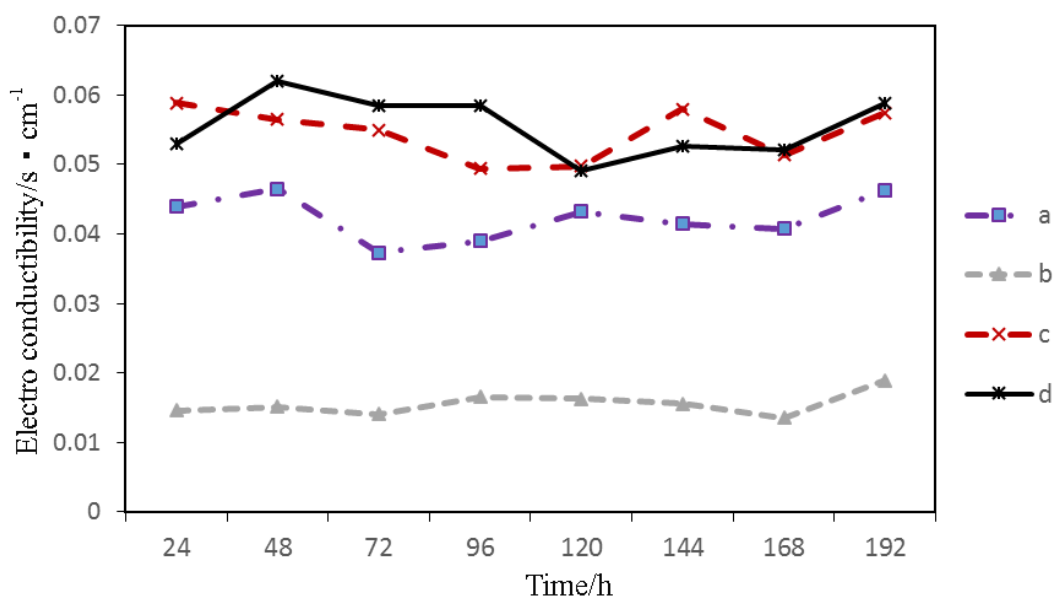

Figure 5Conductivity Curve

(a) SPEEK（b） SPEEK;PHNT =6:4

(c) SPEEK;PHNT;HPW=6:1:3)（d） SPEEK:PHNT:HPW=5:1.25:3.75

\section{Conclusion}

We made theoretical estimates for the performance of the new proton exchange membrane in pre-experimental stage, and estimated the conductivity compared to pure SPEEK proton exchange membrane will increase more than $20 \%$ in the high temperature and low humidity state. Through a series of experiments, the final prepared proton exchange membrane's conductivity compared to pure film SPEEK increases by 36.39\%.

\section{Reference}

[1] Xin Liu, et al. Proton Conductivity Improvement of Sulfonated Poly(ether ether ketone) Nanocomopsite Membranes with Sulfonated Halloysite Nanotubes Prepared via Dopamine-Initiated Atom Transfer Radical Polymerization, J. Membr. Sci. 2016, 504: 206-219.

[2] H. Bai, et al. Enhanced proton conduction of chitosan membrane enabled by halloysite nanotubes bearing sulfonate polyelectrolyte brushes. J. Membr. Sci., 2014, 454: 220-232

[3] B. Zhu, et al. Polydopamine-melanin initiators for surface-initiated ATRP. Polymer, 2011, 52: 2141-2149. 